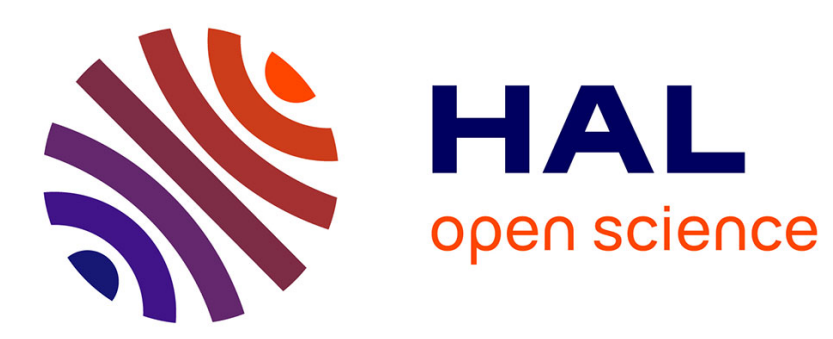

\title{
Decentralized Pricing and the equivalence between Nash and Walrasian equilibrium
}

\author{
Antoine Mandel, Herbert Gintis
}

\section{To cite this version:}

Antoine Mandel, Herbert Gintis. Decentralized Pricing and the equivalence between Nash and Walrasian equilibrium. Journal of Mathematical Economics, 2016, 63, pp.84-92. 10.1016/j.jmateco.2015.12.008 . halshs-01296646

\section{HAL Id: halshs-01296646 \\ https://shs.hal.science/halshs-01296646}

Submitted on 29 Nov 2016

HAL is a multi-disciplinary open access archive for the deposit and dissemination of scientific research documents, whether they are published or not. The documents may come from teaching and research institutions in France or abroad, or from public or private research centers.
L'archive ouverte pluridisciplinaire $\mathbf{H A L}$, est destinée au dépôt et à la diffusion de documents scientifiques de niveau recherche, publiés ou non, émanant des établissements d'enseignement et de recherche français ou étrangers, des laboratoires publics ou privés. 


\title{
Decentralized Pricing and the Equivalence between Nash and Walrasian Equilibrium
}

\author{
Antoine Mandel* and Herbert Gintis ${ }^{\dagger \ddagger}$
}

\begin{abstract}
We introduce, in the standard exchange economy model, market games in which agents use private prices as strategies. We give conditions on the game form that ensure that the only strict Nash equilibria of the game are the competitive equilibria of the underlying economy. This equivalence result has two main corollaries. First, it adds to the evidence that competitive equilibria can be strategically stable even in small economies. Second, it implies that competitive equilibria have good local stability properties under a large class of evolutionary learning dynamics.
\end{abstract}

\section{Introduction}

In his Elements of Pure Economics (see Walras 1874), Walras introduces two descriptions of the price adjustment process in a market economy. On the one hand, he puts forward the tâtonnement as a "theoretical" model. On the other hand, he emphasizes that in practice, the driver of price adjustment is free competition. He characterizes the latter by three conditions (see Dockès and Potier, 2005): free market entry and exit, freedom to set prices and freedom to set production levels.

This paper investigates a game theoretic model of price formation that exhibits the characteristics of free competition in the latter sense. Our key behavioral assumption is that individual agents set prices in a decentralized manner. Our main result is to show the equivalence between Nash and general equilibria in this context. More precisely, we consider a standard exchange economy in which each

* Paris School of Economics, University Paris 1 Panthéon-Sorbonne. Corresponding author, email:antoine.mandel@univ-paris1.fr, tel/fax:+3314407 8271/8301

${ }^{\dagger}$ Santa Fe Institute, 1399 Hyde Park Road, Santa Fe, NM 87501

${ }^{\ddagger}$ The authors are grateful to four anonymous referees for their helpful comments and acknowledge the support of Institute for New Economic Thinking grant INO1200022. Mandel also acknowledges support from the EU-FP7 project IMPRESSION an EU-H2020 project DOLFINS. We dedicate this paper to Hirofumi Uzawa. He will be sorely missed. 
agent has a trade strategy that consists of a vector of private prices for the goods he is endowed with and the goods he consumes. These private prices represent the prices at which the agent is willing to sell the goods he supplies to the market and the maximum prices he is willing to pay for the goods he demands from the market. We consider that agents strategically update their private prices in order to improve their competitive position on different markets. The historically inclined reader will note that this approach closely matches Walras' original description of free competition:

As buyers, traders make their demand by outbidding each other. As sellers, traders make their offers by underbidding each other... The markets that are best organized from the competitive standpoint are those in which... the terms of every exchange are openly announced and an opportunity is given to sellers to lower their prices and to buyers to raise their bids. (Walras 1874, paragraph 41).

We say that a profile of private prices is uniform if all the agents in the economy use the same private prices. We then identify general equilibria of the economy with uniform price profiles for which the common price is a market equilibrium price. Our main result is that these general equilibrium price profiles are the only strict Nash equilibria of the model. This result holds provided that competition is effective in the sense that: (i) when there is positive excess supply in one period, a seller who failed to secure a transaction can gain by slightly undercutting his competitors' prices, (ii) when there is positive excess demand, a buyer who failed to secure a transaction can increase his utility by slightly outbidding his competitors.

The equivalence between general market equilibria and strict Nash equilibria in our model has two main corollaries. First, it adds to the evidence reviewed below that competitive equilibria can be strategically stable even in small economies. Second, it is a well-known result in evolutionary game theory (see Weibull, 1995; Gintis, 2009) that in multi-population games, strict Nash equilibria are the only asymptotically stable points of the replicator dynamic, and more generally of any monotone dynamic. Hence, if we treat an exchange economy as the stage game of an evolutionary process in which each agent's initial inventory is replenished in each period, general equilibria will be the only asymptotically stable states.

Our contribution is related to the large body of literature that focuses on the strategic and evolutionary foundations of general equilibrium. A first strand of literature (see in particular Rubinstein-Wolinsky 1985, Gale 1986a and 1986b, Mclennan and Sonnenschein 1991, and Kunimoto and Serrano 2002) builds on models of bargaining à la Rubinstein (1982) to provide strategic foundations to Walrasian equilibrium. It considers agents who are matched in a sequence of pairwise interactions during which they bargain their endowments and decide whether 
to leave or to stay in the market. The main result in this literature is that agents exit the market when they reach their Walrasian allocation. Hence this literature provides mechanisms that entail the stability of Walrasian allocations. However these mechanisms do not plausibly represent actual market institution and do not address the stability of equilibrium resulting from price dynamics because prices are absent in these models.

In the literature on strategic market games pioneered by Shapley and Shubik (1977) and surveyed in Giraud (2003), institutions are central. Prices are determined at trading posts at which strategically determined nominal demands and real supplies are confronted. Early contributions in this strand of literature have focused on large economies. Their main result is that, as the economy is increasingly replicated, the set of Nash equilibria of the strategic market game converges to the set of general market equilibria of the underlying economy. Our contribution is more closely related to the subsequent literature that has focused on strategic stability in small economies. Peck and Shell (1990) consider a market game model à la Shapley-Shubik in which traders may make arbitrary short sales. This possibility of short sales fosters liquidity and market competition, allowing competitive equilibria to emerge even in small economies. An alternative approach to increase liquidity, pursued by Ghosal and Morelli (2004), is to allow for retrading. Competitive equilibria can then be supported as allocations of the market game in the infinite-time limit. Hence, market games à la Shapley-Shubik yield very similar conclusions to these of the present paper about the strategic stability of competitive equilibria. The key difference is that we consider a decentralized price-formation process in which prices are set by individual agents, whereas in the market game literature prices are set centrally at trading posts. The importance of such differences in the price adjustment mechanism is strongly emphasized in Kumar and Shubik (2004). Additionally, whereas our result is an equivalence, competitive equilibria generally form a strict subset of the set of Nash equilibria of market games. Yet, recent experimental results obtained by Duffy et al. (2011), show that subjects have a tendency to coordinate on efficient Nash equilibria rather than on Pareto inferior ones. Moreover, as the number of subjects participating in the market game increases, the Nash equilibrium that experimental subjects achieve approximates the associated competitive equilibrium of the underlying economy.

These experimental results on market games are complementary to the ones obtained in continuous double-auction experiments (see Smith, 1982; Asparouhova et al., 2011, for surveys of the relevant literature), which give strong support for competitive outcomes. ${ }^{4}$ Our approach is closely related to the theoretical liter-

\footnotetext{
${ }^{4}$ There are also notable exceptions such as Anderson et al. 2004, who show that continuous
} 
ature developed to explore the stability of competitive equilibria in continuous double-auctions (Friedman, 1991; Easley and Ledyard, 1993; Gjerstad and Dickhaut, 1998; Lesourne et al., 2006). First and foremost, private prices are determined by individual traders in both cases. Second, some of the examples of exchange mechanisms considered below are very similar to double auctions. Third, consistently with the evolutionary dimension of our work, the continuous double-auction literature generally consider traders as myopic.

From this last perspective, our approach relates to the evolutionary game theory literature on market dynamics: Vega-Redondo (1997) analyzes the convergence to the Walrasian equilibrium in a Cournot oligopoly where firms update quantities in an evolutionary fashion, Alós-Ferrer et al. (2000) provide an evolutionary model of Bertrand competition, Serrano and Volij (2008) study the stability of Walrasian equilibrium in markets for indivisible goods, and several contributions (Mandel and Botta, 2009; Kim and Wong, 2011; Mandel and Gintis, 2014), investigate evolutionary dynamics in specific exchange economies. Though less precise on dynamical aspects, our contribution provides results that are more generic than those of the existing literature as it applies to a broad class of exchange economies.

The rest of the paper is organized as follows. Section 2 introduces our model economy. Section 3 defines a class of market games based on private prices in this economy and gives necessary conditions for the stability of equilibrium. Section 4 analyzes in greater detail the necessary conditions for competition to entail a stable price adjustment process. In section 5, we illustrate numerically the extension of our results to Markovian price adjustment processes. Section 6 concludes.

\section{The Walrasian Economy}

We consider an economy with a finite set of goods $G=\{1, \ldots, n\}$, and a finite set of agents $A=\{1, \ldots, m\}$. Each agent $i \in A$ has consumption set $\mathcal{X}=\mathbf{R}_{+}^{n}$, a utility function $u_{i}: \mathcal{X} \rightarrow \mathbf{R}_{+}$and an initial endowment $e_{i}=\left(e_{i 1}, \ldots, e_{i n}\right) \in \mathcal{X}$. We denote this economy by $\mathcal{E}(u, e)$, where $u=\left(u_{1}, \ldots, u_{m}\right)$ and $e=\left(e_{1}, \ldots, e_{m}\right)$.

Our subsequent analysis will be greatly simplified in the case where for each agent one can distinguish consumption goods, those the agent consumes, from endowment goods, those with which the agent is endowed. ${ }^{5}$ For agent $i \in A$, the set of endowment goods is given by $E_{i}=\left\{g \in G \mid e_{i g}>0\right\}$. The set of consumption goods is denoted by $C_{i}$ and characterized by the following assumption.

double auction can yield tâtonnement-like orbits in the Scarf economy.

${ }^{5}$ This setting is sometimes referred to as "buy-or-sell game" in the economic litterature and is known under the name of Fisher economies in the algorithmic game theory literature. 
Assumption 1 (Goods). For all $i \in A$, there exists $C_{i} \subset G$ such that $C_{i} \cap E_{i}=\emptyset$ and for all $x, y \in \mathcal{X}$ one has: $\left(\forall g \in C_{i}, x_{g}=y_{g}\right) \Rightarrow u_{i}(x)=u_{i}(y)$.

Accordingly, we define the set of buyers of good $g$ as $B_{g}=\left\{i \in \mathcal{A} \mid g \in C_{i}\right\}$, and the set of sellers of good $g$ as $S_{g}=\left\{i \in \mathcal{A} \mid g \in E_{i}\right\}$.

The following concepts are standard in the analysis of exchange economies.

- An allocation $x \in \mathcal{X}^{m}$ of goods is feasible if for all $g \in G$ :

$$
\sum_{i=1}^{m} x_{i g} \leq \sum_{i=1}^{m} e_{i g} .
$$

We write $\mathcal{A}\left(e_{1}, \ldots, e_{m}\right)=\mathcal{A}(e) \subset \mathcal{X}^{m}$ for the set of feasible allocations.

- The price space is $\mathcal{P}=\mathbf{R}_{+}^{n-1} \times\{1\}$ and good $n$ is called the numéraire good.

- The demand of agent $i$ is a mapping $d_{i}: \mathcal{P} \times \mathbf{R}_{+} \rightarrow \mathcal{X}$ that associates to a price $p \in \mathcal{P}$ and an income $w \in \mathbf{R}_{+}$the utility-maximizing individual allocations satisfying the budget constraint:

$$
d_{i}(p, w)=\operatorname{argmax}_{\left\{x_{i} \in \mathcal{X} \mid p \cdot x_{i} \leq w\right\}} u_{i}\left(x_{i}\right) .
$$

- A feasible allocation $x \in \mathcal{A}(e)$ is an equilibrium allocation if there exists a price $p \in \mathcal{P}$ such that for all $i, x_{i} \in d_{i}\left(p, p \cdot e_{i}\right)$. The price $p$ is then called an equilibrium price. We denote the set of equilibrium prices by $\mathcal{P}_{\text {equi }}(u, e)$.

- A feasible allocation $x \in \mathcal{A}(e)$ and a price $p \in \mathcal{P}$ form a quasi-equilibrium if for all $i$ and for all $y \in \mathcal{X}^{m}, u_{i}\left(y_{i}\right)>u_{i}\left(x_{i}\right)$ implies $p \cdot y_{i}>p \cdot x_{i}$.

Our focus being on the stability of equilibrium, we place ourselves throughout the paper in a setting where sufficient conditions for the existence of an equilibrium hold. First, we assume that utility functions satisfy the following standard conditions.

Assumption 2 (Utility). For all $i \in A, u_{i}$ is continuous, its restriction to $R^{C_{i}}$ is strictly concave and locally non-satiated and $u_{i}(x)>0$ only if for all $g \in C_{i}$, $x_{g}>0$.

This assumption suffices to guarantee the existence of a quasi-equilibrium. Moreover, the strict concavity assumption implies that demand mappings are singlevalued, which proves useful below. The last condition implies that the consumption 
of each consumption good is necessary and is fairly standard in general equilibrium theory (see Mas-Collel 1989).

Second, to ensure that every quasi-equilibrium is an equilibrium allocation, it suffices to assume that at a quasi-equilibrium, agents do not receive the minimal possible income (see Hammond, 1993; Florenzano, 2005). This condition is satisfied when all initial endowments are in the interior of the consumption set, as well as in settings with corner endowments such as those investigated in Scarf (1960) and Gintis (2007). Formally, the assumption can be stated as follows.

Assumption 3 (Income). For every quasi-equilibrium $(p, x)$, there exists an allocation $y$ such that $p \cdot x_{i}>p \cdot y_{i}$ for all $i \in A$.

The Utility Assumption (2) and the Income Assumption (3) then imply that the economy $\mathcal{E}(u, e)$ has at least one equilibrium (see Florenzano, 2005). We shall assume they hold throughout the paper and will further restrict attention to the generic case where the set $\mathcal{P}_{\text {equi }}$ of equilibria is finite (see Balasko 2009).

\section{Exchange Economies with Private Prices}

\subsection{The Game Form}

We represent the exchange process in the economy $\mathcal{E}(u, e)$ as a game in which agents set prices in a decentralized manner and the resulting distribution of prices determines the agents' allocations. More precisely, we consider that each agent $i \in A$ has a strategy consisting of a private price vector $\pi_{i} \in \mathcal{P}_{i}=\mathbf{R}_{+}^{G_{i}}$, where $G_{i}=C_{i} \cup E_{i}$. This private price vector represents the prices at which he is willing to sell the goods he supplies to the market and the maximum prices he is willing to pay for the goods he demands. We then associate to the economy $\mathcal{E}(u, e)$ the game $\mathcal{G}(u, e, \xi)$ such that:

- Each agent $i \in A$ has the set of prices $\mathcal{P}_{i}$ as strategy set, so that the set of strategy profiles for the game is $\Pi=\mathcal{P}_{1} \times \cdots \times \mathcal{P}_{m}$.

- The game form is defined by an exchange mechanism $\xi: \Pi \rightarrow \mathcal{A}(e)$ that associates to a profile of private prices $\pi \in \Pi$ a feasible allocation $\xi(\pi)=$ $\left(\xi_{1}(\pi), \ldots, \xi_{m}(\pi)\right) \in \mathcal{A}(e)$.

- The payoff of player $i$ is $u_{i}\left(\xi_{i}(\pi)\right)$.

Many exchange processes can be represented in this way including central clearing systems such as double auctions, simultaneous multilateral exchanges as usually considered in general equilibrium models with out-of-equilibrium features 
such as Grandmont (1977), and sequential bilateral exchanges such as those considered by Gintis $(2007,2012)$.

\subsection{Definitions and Notation}

In our analysis of the game $\mathcal{G}(u, e, \xi)$, we shall use the following notions:

- A strategy profile $\pi \in \Pi$ is $p$-uniform for price $p \in \mathcal{P}$ if, for all $i \in A$ and $g \in G_{i}$, one has $\pi_{i g}=p_{g}$.

- A $p$-uniform strategy profile $\pi$ is a general equilibrium price profile if $p \in$ $\mathcal{P}_{\text {equi }}(u, e)$.

- A strategy profile $\pi$ is $p$-seller uniform if for all $g \in G$ and all $i \in S_{g}$, we have $\pi_{i g}=p_{g}$.

- For $\pi \in \Pi, i \in A, g \in G$, and $q_{i g} \in \mathbb{R}_{+}$we denote by $\left(\pi_{-i g}, q_{i g}\right)$ the strategy profile $\pi^{\prime}$ such that $\pi_{i g}^{\prime}=q_{i g}$ and for $(j, h) \in A \times G_{j},(j, h) \neq(i, g)$, we have $\pi_{j h}^{\prime}=\pi_{j h}$.

- For $\pi \in \Pi, i \in A$, and $p \in \mathcal{P}_{i}$ we denote by $\left(\pi_{-i}, p\right)$ the strategy profile $\pi^{\prime}$ such that $\pi_{i}^{\prime}=p$ and for $j \in A, j \neq i, \pi_{j}^{\prime}=\pi_{j}$.

\subsection{Strict Nash Equilibria and Stability}

In the following, we will specify a broad class of exchange processes $\xi$ for which general equilibrium price profiles coincide with strict Nash equilibria of the game $\mathcal{G}(u, e, \xi)$. These are defined as follows:

Definition 1. A strategy profile $\pi \in \Pi$ is a strict Nash equilibrium of $\mathcal{G}(u, e, \xi)$ if for all $i \in A$ and all $p \in \mathcal{P}_{i}$ such that $p \neq \pi_{i}$, one has:

$$
u_{i}\left(\xi_{i}(\pi)\right)>u_{i}\left(\xi_{i}\left(\pi_{-i}, p\right)\right) .
$$

Our focus on strict Nash equilibrium is motivated by its central role in the theory of learning in games. In evolutionary game theory (see Weibull, 1995; Gintis, 2009) most of the learning processes, including the replicator dynamic, are monotone dynamics in the sense that players tend to switch from worse to better performing strategies. A key result is that for monotone dynamics, strict Nash equilibria of the stage game are the only asymptotically stable states (see Weibull 1995). Hence, if they can be identified with strict Nash equilibria of the game $\mathcal{G}(u, e, \xi)$, general market equilibria of the economy $\mathcal{E}(u, e)$ will be the only asymptotically 
stable states for any monotone dynamic. In particular, this sheds light on the convergence to general equilibrium observed in the simulations in Gintis (2007) and Gintis (2012) where agents update their prices by imitating those of more successful peers. Indeed there is a well-established relationship between these stochastic imitation models and the replicator dynamic (see Benaim and Weibull, 2003).

Note however that observing other agents' payoffs is not a necessary condition for the asymptotic stability of strict Nash equilibria. For example, Ianni (2013) shows that strict Nash equilibria are asymptotically stable states for reinforcement learning processes, for which price updates depends only on an agent's own experience. More generally, through the replicator dynamic, the stability of strict Nash equilibria extend to a broad class of learning dynamics. Indeed, the replicator forms the backbone of many learning processes. In particular, Hopkins (2002) shows that stochastic fictitious play and reinforcement learning with experimentation can both be written as a perturbed form of the evolutionary replicator dynamic and Shalizi (2009) shows that rather mild conditions ensure an equivalence between Bayesian updating and the replicator dynamic.

Beyond dynamic stability, the equivalence between strict Nash and general equilibria will guarantee that general market equilibria are risk-dominant because they are the only strict equilibria.

\subsection{An axiomatic characterization of stable exchange processes}

To analyze the relationships between the behavior of the agents in the economy $\mathcal{E}(u, e)$ and the game $\mathcal{G}(u, e, \xi)$, we first extend the notion of demand to our framework with private prices. In this setting, an agent's demand may depend not only on his own private prices, but also on the prices posted by his potential suppliers. For instance, a buyer of good $g$ may sample a subset of sellers of good $g$ who post their selling prices, and choose his demand for $g$ based on the information received. To subsume all possible cases under a common framework, we assume that each agent $i$ has an extended demand function $\delta_{i}: \Pi \times \mathbf{R}_{+} \rightarrow \mathcal{X}$, that associates to a strategy profile $\pi \in \Pi$ and an income $w \in \mathbb{R}_{+}$, a demand $\delta_{i}(\pi, w)$.

Given that our analysis takes place out of equilibrium, realized income cannot be determined ex ante as it depends on the particular trades an agent succeeds in executing. Rather, we assume that agents choose strategies based on expected income. The income expected by an agent is also prima facie a function of the complete distribution of private prices. In particular, the expected income of an agent $g$ can depend on the prices set by his competitors. Hence, we represent the income expected by seller $i \in S_{g}$ on good $g$ market by a function $w_{i g}: \Pi \rightarrow \mathbb{R}_{+}$ and his total expected income by $w_{i}=\sum_{g \in E_{i}} w_{i g}$. 
Therefore, when the prevailing strategy profile is $\pi$, we refer to $\delta_{i}(\pi, w(\pi))$ as the extended demand (or simply the demand if there is no ambiguity) of agent $i$. We say there is excess demand or excess supply for good $g$ at a strategy profile $\pi \in \Pi$ if $\sum_{i \in B_{g}} \delta_{i g}(\pi, w(\pi))>\sum_{j \in S_{g}} e_{j g}$, or $\sum_{i \in B_{g}} \delta_{i g}(\pi, w(\pi))<\sum_{j \in S_{g}} e_{j g}$, respectively. When the former inequalities are weak, we say respectively that there is weak excess demand or weak excess supply.

On the basis of this notion of extended demand, we can relate the behavior of agents in the private price game and in the general equilibrium economy. We shall first assume that when the strategy profile is uniform and the extended demand and income functions coincide with their general-equilibrium counterparts:

Assumption 4 (Price Consistency). For all agents $i$ and all $\pi \in \Pi$,

1. $w_{i h}(\pi) \leq \pi_{i h} e_{i h}$, with equality when $\pi$ is p-uniform;

2. If $\pi$ is p-uniform, then $\delta_{i}(\pi, \cdot)=d_{i}(p, \cdot)$.

Second, we shall assume that at a general equilibrium price profile, the corresponding equilibrium allocation prevails.

Assumption 5 (Equilibrium). If $\pi \in \Pi$ is a p-uniform general equilibrium price profile, then for all agents $i$ and all prices $q \in \mathcal{P}_{i}$,

1. $\xi_{i}(\pi)=d_{i}\left(p, p \cdot e_{i}\right)$.

2. If $u_{i}\left(\xi_{i}\left(\pi_{-i}, q\right)\right) \geq u_{i}\left(d_{i}\left(p, p \cdot e_{i}\right)\right)$ then either $q \leq p_{i}$ or $w_{i}\left(\pi_{-i}, q\right) \geq p \cdot e_{i}$.

The first part of the assumption is a minimal efficiency requirement on the exchange process without which no relation could be established between the equilibrium strategy profiles of $\mathcal{G}(u, e, \xi)$ and the equilibria of the economy $\mathcal{E}(u, e)$. The second part of the assumption ensures that, at a general equilibrium price profile, the exchange mechanism is consistent with the agent's demand. Namely, there can be a profitable deviation from a general equilibrium price profile only if the budget set increases through an increase of income or a decrease in prices.

We now turn to the constraints private prices place on trading out of equilibrium. A private price represents the maximum price a buyer is willing to pay for one unit of good and conversely the minimum price a seller is willing to accept for one unit of good. Hence, the least constraint one can impose on the trading mechanism is that buyers whose private price for a good $g$ is below this of the lowest seller price do not receive any allocation of good $g$. Conversely sellers whose private price for good $g$ is above the highest buyer price cannot raise any income on the market for good $g$. That is, we have: 
Assumption 6 (Price Compatibility). For any strategy profile $\pi \in \Pi$ and any good g, we have:

1. For any buyer $i \in B_{g}$, iffor all sellers $j \in S_{g}, \pi_{j g}>\pi_{i g}$, then $\xi_{i g}(\pi)=0$.

2. For any seller $j \in S_{g}$, iffor all buyers $i \in B_{g}, \pi_{i g}<\pi_{j g}$, then $w_{j g}(\pi)=0$.

We expect competition to be the key force leading to equilibrium in a decentralized economy. Accordingly, we shall assume that the exchange mechanism implements some form of competition both among buyers and among sellers. To account for competition among buyers, we assume that when there is positive excess demand for good $g$, a buyer has incentive to outbid his competitors.

Assumption 7 (Buyer Competition). Let $\pi \in \Pi$ be such that there is excess demand for good $g$. Then there exists a buyer $i \in B_{g}$ and a price $q_{i g}>\pi_{i g}$ such that $u_{i}\left(\xi_{i}\left(\pi_{-i g}, q_{i g}\right)\right) \geq u_{i}\left(\xi_{i}(\pi)\right)$.

For competition among sellers, we assume that when there is excess supply for good $g$, a seller of good $g$ can raise his market share and hence his income by undercutting his competitors. That is:

Assumption 8 (Seller Competition). Let $\pi \in \Pi$ be such that there is weak excess supply for good $g$ and there are sellers $i, j \in S_{g}$ with $\pi_{i g}>\pi_{j g}$. Then there is a price $q_{i g}<\pi_{i g}$ such that $w_{j g}\left(\pi_{-i g}, q_{i g}\right) \geq w_{j g}(\pi)$.

The Seller Competition Assumption (8) will have an actual impact on behavior only if, all other things being equal, an agent's allocation is a non-decreasing function of income. Therefore we posit:

Assumption 9 (Income Monotonicity). Let $\pi, \pi^{\prime} \in \Pi$, and suppose that for some agent $i$, we have $\pi_{-i}=\pi_{-i}^{\prime}$ and for all $g \in C_{i}, \pi_{i g}=\pi_{i g}^{\prime}$. Then if $w_{i}(\pi) \geq w_{i}\left(\pi^{\prime}\right)$, we have $u_{i}\left(\xi_{i}(\pi)\right) \geq u_{i}\left(\xi_{i}\left(\pi^{\prime}\right)\right)$.

Last, in order to prevent buyers from indefinitely increasing their prices, one should assume that there is some form of counterweight to the Buyer Competition Assumption (7). That is, in case of excess supply, buyers incur losses if their private price for a good is above the highest selling price (for instance, because price dispersion hampers coordination).

Assumption 10 (Homogeneous Pricing). Let $\pi \in \Pi$ be such that there is weak excess supply for good $g$ and for some buyer $i \in B_{g}, \pi_{i g}>\max _{j \in S_{g}} \pi_{j g}$. Then there is a price $q_{i g}<\pi_{i g}$ such that $u_{i}\left(\xi_{i}\left(\pi_{-i g}, q_{i g}\right)\right) \geq u_{i}(\xi(\pi))$. 
To sum up, we have on the one hand structural assumptions (4) to (6) that ensure the consistency of the exchange game with the underlying economy and on the other hand behavioral assumptions (7) to (10) that ensure the exchange mechanism implements some form of competition in the Walrasian sense. These conditions suffice to ensure the strategic stability of Walrasian equilibrium. Namely, we have:

Theorem 1. Let $\mathcal{E}(u, e)$ be an economy and $\xi$ an exchange mechanism such that Assumptions (2) to (10) hold. Then a strategy profile $\pi$ is a strict Nash equilibrium of the game $\mathcal{G}(u, e, \xi)$ if and only if $\pi$ is p-uniform for some general market equilibrium price vector $p$.

Proof: Suppose $\pi$ is a $p$-uniform general equilibrium price profile. We must show that $\pi$ is a strict Nash equilibrium. According to the Equilibrium Assumption (5), for all agents $i, \xi_{i}(\pi)=d_{i}\left(p, p \cdot e_{i}\right)$. Suppose agent $i$ deviates to a price vector $q \neq p_{i}$.

For $g \in E_{i}$, we have:

- If $q_{g}>p_{g}$, then according to the Price Compatibility Assumption (6), we have $w_{i g}\left(\pi_{-i}, q\right)=0$.

- If $q_{g}<p_{g}$, then according to the Price Consistency Assumption (4), we have $w_{i g}\left(\pi_{-i}, q\right) \leq q_{g} e_{i g}<p_{g} e_{i g}=w_{i g}(\pi)$.

Hence we have

$$
w_{i}\left(q, \pi_{-i}\right) \begin{cases}<w_{i}(\pi) & \text { if } q_{g} \neq p_{g} \text { for some } g \in E_{i} \\ =w_{i}(\pi) & \text { otherwise }\end{cases}
$$

Then, for $g \in C_{i}$, we have three possibilities:

- Case 1: There is some $g \in C_{i}$ such that $q_{g}<p_{g}$. Then according to the Price Compatibility Assumption (6), we have $\xi_{i g}\left(\pi_{-i}, q\right)=0$. This implies, by the Goods Assumption (1), that $u_{i}\left(\xi_{i}\left(\pi_{-i}, q\right)\right)<u_{i}\left(d_{i}\left(p, p \cdot e_{i}\right)\right)=u_{i}\left(\xi_{i}(\pi)\right)$.

- Case 2: For all $g \in C_{i}, q_{g} \geq p_{g}$ with the inequality being strict for some $g$. As moreover, according to equation (1), we have $w_{i}\left(q, \pi_{-i}\right) \leq w_{i}(\pi)$, it is clear from the Equilibrium Assumption (5) that $u_{i}\left(\xi_{i}\left(\pi_{-i}, q\right)\right)<u_{i}\left(\xi_{i}(\pi)\right)$.

- Case 3: $q_{g}=p_{q}$ for all $g \in C_{i}$. Then, as $q \neq p$, there is a good $h \in E_{i}$ such that $q_{h} \neq p_{h}$. This implies according to (1) that $w_{i}\left(\pi_{-i}, q\right)<w_{i}(\pi)$ and hence, using the Equilibrium Assumption (5), that $u_{i}\left(\xi_{i}\left(\pi_{-i}, q\right)\right)<$ $u_{i}\left(\xi_{i}(\pi)\right)$. 
This proves that every general equilibrium price profile is a strict Nash equilibrium.

Conversely, let us show that if $\pi \in \Pi$ is not a general equilibrium price profile then $\pi$ is not a strict Nash equilibrium of $\mathcal{G}(u, e, \xi)$. To prove this result by contradiction, suppose $\pi$ were a strict Nash equilibrium.

- According to the Buyer Competition Assumption (7), if there is excess demand for good $g$ for the strategy profile $\pi$, then there is a buyer $i \in B_{g}$ and a price $q_{i g}>\pi_{i g}$ such that $u_{i}\left(\xi_{i}\left(\pi_{-i g}, q\right)\right) \geq u_{i}(\xi(\pi))$ and hence $\pi$ cannot be a strict Nash equilibrium. By contraposition, this implies that there can be no excess demand for any good at the price profile $\pi$.

- Let us then remark that if $\pi$ is a $p$-uniform strategy profile, then by the Price Consistency Assumption (4) we have that for all $i \in A, \delta_{i}\left(\pi, w_{i}(\pi)\right)=$ $d_{i}\left(p, p \cdot e_{i}\right)$. Walras Law then implies that, unless $p$ is an equilibrium price, there is excess demand for at least one good. This would contradict the preceding point. If the only good for which there is excess demand is the numéraire good, there must be excess supply for another good and a similar argument applies.

Hence $\pi$ is not a $p$-uniform strategy profile.

- We now have that $\pi$ is not $p$-uniform and that there is weak excess supply for every good. We can then distinguish the following cases: ${ }^{6}$

- Case 1: $\pi$ is not $p$-seller uniform for any price $p \in \mathcal{P}$. There then exists a good $g$ and sellers $i, j \in S_{g}$ such that there is weak excess supply for good $g$ and $\pi_{j g}<\pi_{i g}$. Then by the Seller Competition Assumption (8), there exists $q_{i g}<\pi_{i g}$ such that $w_{i g}\left(\pi_{-i g}, q_{i g}\right) \geq w_{i g}(\pi)$. Therefore, using the Income Monotonicity Assumption (9), we have $u_{i}\left(\xi_{i}\left(\pi_{-i g}, q_{i g}\right)\right) \geq u_{i}\left(\xi_{i}(\pi)\right)$ and $\pi$ is not a strict Nash equilibrium.

- Case 2: $\pi$ is $p$-seller uniform for some $p \in \mathcal{P}$. We then have:

* Case 2a: There exists $i \in B_{g}$ such that $\pi_{i g}<p_{g}$. Then by the Price compatibility Assumption (6), we have $\xi_{i g}(\pi)=0$ and hence $u_{i}\left(\xi_{i}(\pi)\right)=0$. Therefore, it is clearly profitable for agent $i$ to shift to price $p_{g}$ and $\pi$ is not a strict Nash equilibrium.

* Case 2b: For all $i \in B_{g}, \pi_{i g} \geq p_{g}$ and, because $\pi$ is not uniform, for at least one $k \in B_{g}$, one has $\pi_{k g}>p_{g}$. As there is

\footnotetext{
${ }^{6}$ In each of the following cases, good $g$ cannot be the numéraire good because there exist two different prices for good $g$ in the population.
} 
weak excess supply for good $g$, we can apply the Homogeneous Pricing Assumption (10). Hence there exists $q<\pi_{k g}$ such that $u_{i}\left(\xi_{i}\left(\pi_{-i g}, q\right)\right) \geq u_{i}(\xi(\pi))$ and $\pi$ is not a strict Nash equilibrium.

We have thus shown that whenever $\pi$ is not a $p$-uniform general equilibrium strategy profile, it is not a strict Nash equilibrium.

\section{Discussion and Examples}

In this section, we first illustrate why the conditions put forward in Theorem 1 are necessary to obtain stability results and then provide examples of exchange mechanisms satisfying the required conditions.

\subsection{Convergence and Efficiency}

It is clear that at a non-equilibrium $p$-uniform strategy profile, agents have an incentive to deviate. Indeed, Walras Law implies there is excess demand for one good, so that buyers are rationed and hence have an incentive to increase their prices slightly to gain a competitive edge over their competitors. Conversely, there is excess supply for at least one good so that sellers have an incentive to lower their prices to gain a competitive edge. These deviations are profitable in our framework with private prices because they generally leave the demand and the supply of other agents unchanged. This is in contrast with the tâtonnement process where any variation of the public price changes the complete profile of supply and demand.

If it is relatively straightforward to show that non-equilibrium uniform price profiles cannot be stable (Price Consistency Assumption (4) and Buyer Competition Assumption (7) would suffice in this respect), the key questions are how do agents coordinate on a common price and why are there no strict Nash equilibria where the market is segmented in sub-markets using different prices? As the following example shows, the key to avoiding this failure is to assume some form of inefficiency of the allocation mechanism out of equilibrium.

Consider, in an otherwise arbitrary economy, the market for a good with two buyers $\left(b_{1}\right.$ and $\left.b_{2}\right)$ and two sellers $\left(s_{1}\right.$ and $\left.s_{2}\right)$. Assume that the private price for the good of $s_{1}$ and $b_{1}$ is 1 , and this of $s_{2}$ and $b_{2}$ is 2 . This implies, assuming the Price Compatibility Assumption (6) holds, that $s_{1}$ can sell to both $b_{1}$ and $b_{2}$ while $s_{2}$ can only sell to $b_{2}$. Assume further that $s_{1}$ has an endowment of 20 units and $s_{2}$ an endowment of 6 units while $b_{1}$ demands 20 units and $b_{2} 5$ units at the prevailing price. There is thus excess supply. Assume then that the exchange mechanism is efficient in the sense that as large a share of supply as possible is allocated given the price compatibility constraints. We then have: 
- At the prevailing price, $s_{1}$ supplies 20 units at price 1 to $b_{1}$ and $s_{2} 5$ units at price 2 to $b_{2}$.

- If $s_{1}$ increases his price, he loses the demand from $b_{1}$ and the sole demand from $b_{2}$ must be allocated between $s_{1}$ and $s_{2}$. The income from $s_{1}$ then necessarily decreases.

- If $s_{2}$ increases his price, he drives himself out of the market and has income 0 .

- If $s_{1}$ decreases his price, he still supplies 20 units to $b_{1}$ but at a lower price and hence his income decreases.

- If $s_{2}$ decreases his price to a price below 1 , his income necessarily decreases.

- If $s_{2}$ decreases his price to a price above 1 , he still supplies uniquely to $b_{2}$ and decreases his income unless the income-elasticity of $b_{2}$ is greater than unity.

Hence, unless the income-elasticity of $b_{2}$ is greater than one, neither buyer has an incentive to change its price, the Seller Competition Assumption (8) fails to hold and there exists a stable non-uniform strategy profile. This failure stems from the fact that we have assumed the exchange mechanism to be efficient. In other words, if the economy functions efficiently with heterogeneous prices, it can settle in states where "multiple equilibria" coincide in different clusters on the same market and agents have no incentive to change their prices.

However, assuming that the economy organizes efficiently despite heterogeneous prices seems behaviorally inconsistent. In the previous example, it is implausible that $b_{2}$ agree to trade with $s_{2}$ despite the presence of a much cheaper seller, unless there are information imperfections and $b_{2}$ does not know about $s_{1}$. In the proof of Theorem 1, the Seller Competition Assumption (8), the Income Monotonicity Assumption (9) and the Homogeneous Pricing Assumption (10) implicitly account for these behavioral aspects. In particular, as illustrated by the examples below, they imply that the exchange mechanism cannot be efficient out of equilibrium.

\subsection{A Tâtonnement-like Exchange Mechanism}

For every economy without no-trade equilibrium, a trivial example of exchange mechanism satisfying assumptions (4) to (10) is the tâtonnement-like mechanism 
where no trade takes place unless excess demand is zero. That is the mechanism defined by:

$$
\xi_{i}^{\text {tat }}(\pi)= \begin{cases}d_{i}\left(p, p \cdot e_{i}\right) & \text { if } \pi \text { is a } p \text {-uniform equilibrium profile } \\ e_{i} & \text { otherwise. }\end{cases}
$$

Note that in this setting, extended demand can be defined for $(\pi, w) \in \Pi \times \mathbb{R}_{+}$by $\delta_{i}(\pi, w)=d_{i}\left(\pi_{i}, w\right)$ and expected income as

$$
w_{i g}(\pi)= \begin{cases}\pi_{i g} \cdot e_{i g} & \text { if } \pi \text { is a } p \text {-uniform strategy profile } \\ 0 & \text { otherwise. }\end{cases}
$$

It is then straightforward to check that assumptions (4) to (10) are satisfied. Therefore, one has:

Corollary 1. If $\mathcal{E}(u, e)$ is an economy without no-trade equilibrium, then $\pi$ is a strict Nash equilibrium of the game $\mathcal{G}\left(u, e, \xi^{\text {tat }}\right)$ if and only if it is a general market equilibrium price profile.

One should notice that this specific result holds independently of assumptions (1), (2) or (3) and hence proves the strategic stability of general equilibrium for any economy without no-trade equilibria. However, in relation to the discussion in Section 4.1, we shall emphasize that the tâtonnement process assumes some extreme form of inefficiency out of equilibrium: no trade takes place. Hence this process would be ruled-out if we assumed some form of minimal efficiency such as considering that when there exist Pareto-improving trades a non-zero fraction of the gains is indeed seized by the exchange mechanism.

\subsection{Price-based Priority}

We now explore exchange mechanisms where competition between agents is implemented through constraints on the access to the market.

On the supply side, let us first assume that only the lowest price sellers have access to the market. That is, if the minimal price for good $g$ is denoted by $\hat{\pi}_{g}=$ $\min _{j \in S_{g}} \pi_{j g}$, the income of agent $i$ on the market for good $g$ is given by:

$$
w_{i g}(\pi)= \begin{cases}\pi_{i g} \cdot e_{i g} & \text { if } \pi_{i g}=\hat{\pi}_{g} \\ 0 & \text { otherwise. }\end{cases}
$$

Accordingly, we assume that demand is based on the prices of the lowest price sellers, that is for all $(\pi, w) \in \pi \times \mathbb{R}_{+}$, we have

$$
\delta_{i}(\pi, w)=d_{i}(\hat{\pi}, w),
$$


where $\hat{\pi}=\left(\hat{\pi}_{1}, \ldots, \hat{\pi}_{n}\right)$.

We assume that competition between buyers takes the form of the highest bidding buyers having priority access to the market. That is, for each good, demands are ordered by decreasing buying price and are fulfilled sequentially until the admissible supply (this from the lowest price sellers) is exhausted. More precisely, we consider the exchange mechanism $\xi^{\text {prio }}$ is defined by the following algorithm.

For $g=1$ to $n\{$

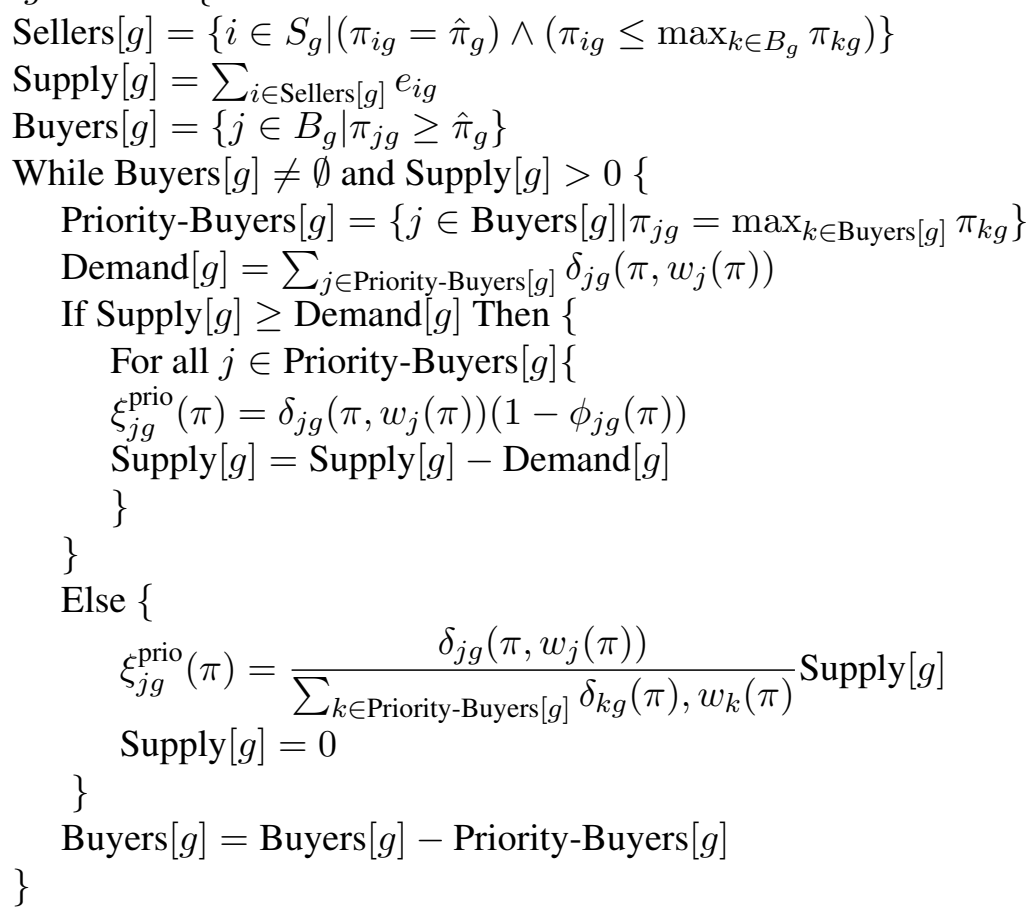

Here $\phi_{j g}: \Pi \rightarrow[0,1]$ is a penalty function such that $\phi_{j g}(\pi)>0$ if and only if there is excess supply of good $g$ and $\pi_{j g}>\hat{\pi}_{g}$. As stressed in the discussion of the Homogeneous Pricing Assumption (10), this penalty is a counterweight to the Buyer competition Assumption (7) that prevents buyers from indefinitely increasing their prices in case of excess supply.

In this setting, the Price Consistency Assumption (4) and the Price Comparability Assumption (6) are satisfied by construction. The first part of the Equilibrium Assumption (5) is trivially verified and the second part holds because we have by construction for all agents $i$ and all $\pi \in \Pi, \xi_{i}^{\text {prio }}(\pi) \leq d_{i}\left(\hat{\pi}, \hat{\pi} \cdot e_{i}\right)\left(1-\phi_{j g}(\pi)\right)$. The Buyer Competition Assumption (7) holds because if there is excess demand a buyer who raises his buying price increases his priority without modifying his demand (because the demand is computed at the sellers' prices $\hat{\pi}$.) The Homoge- 
neous Pricing Assumption (10) holds because in case of excess supply, a buyer who decreases his price so as to approach the sellers' price does not modify his demand, which is computed at sellers' price, and is not harmed by the potential decrease of his priority order because there is excess supply. The Seller Competition Assumption (8) clearly holds because a seller who is above the minimal selling price for a good does not execute any exchanges on that good market and hence cannot decrease his income by decreasing his price. Finally, the Income Monotonicity Assumption (9) clearly holds if we assume there is no inferior good in the economy. Otherwise, in the particular case where there is excess supply of inferior goods and excess demand for normal goods, it could be that an agent who increases his income decreases his utility when he shifts his demand from inferior to normal goods, which are rationed. One therefore has:

Corollary 2. If $\mathcal{E}(u, e)$ is an economy with no inferior goods and satisfying assumptions (1) to (3), then $\pi$ is a strict Nash equilibrium of the game $\mathcal{G}\left(u, e, \xi^{\text {prio }}\right)$ if and only if it is a general market equilibrium price profile.

In order to dispense of the no inferior-good condition, one could consider that the agent chooses the optimal expenditure level given the rationing constraints he faces. With respect to the relation between inefficiency and stability discussed in Section (4.1), one can notice that the exchange mechanism $\xi^{\text {prio }}$ is inefficient out of equilibrium as only the lowest price sellers have access to the market, though there could be additional matches between high bidding buyers and sellers. This inefficiency has a quite intuitive behavioral justification: agents are reluctant to purchase goods at a high price when they are aware that other trades take place at a lower price. This interpretation is valid, stricto sensu, only if one presumes that each agent observes the complete set of prices. However, the properties of the exchange mechanism $\xi^{\text {prio }}$ can easily be extended to settings with weaker informational requirements. For example, one could consider that all sellers but the most expensive ones have access to the market and that a buyer determines his prices on the basis of the admissible seller whose private price is the closest to his. Alternatively, one could consider that buyers and sellers are connected through a network and that a buyer buys only from the lowest bidding seller to whom he is connected.

\subsection{Bargaining-like Mechanisms}

An alternative interpretation of Theorem 1 can be obtained through the prism of the bargaining literature. Indeed, one can consider that a profile of private prices defines a restricted set of feasible allocations and interpret the choice of an allocation mechanism as the choice of a solution to the bargaining problem in this set. In this 
setting, Theorem 1 can be interpreted as stating that agents have an incentive on the one hand to agree on a common price in order to increase the size of the bargaining set, and on the other hand to deviate from the common price by underbidding or overbidding their competitors when there is excess supply or demand.

The incentive to agree on a common price is related to the constraints private prices put on trading possibilities. In this respect, the least stringent condition we can assume is to consider that access to the market is restricted to traders that have a possibility to find a trade partner: buyers whose private price for a good is above the lowest private price of sellers, and conversely sellers whose private price for a good is below the highest private price of buyers. Accordingly, given a private price vector $\pi$, we define the set of acceptable buyers as:

$$
\mathcal{B}_{g}(\pi)=\left\{i \in B_{g} \mid \pi_{i g} \geq \min _{j \in S_{g}} \pi_{j g}\right\},
$$

and the set of acceptable sellers as:

$$
\mathcal{S}_{g}(\pi)=\left\{i \in S_{g} \mid\left(\pi_{j g} \leq \max _{i \in B_{g}} \pi_{i g}\right) \wedge\left(\pi_{j g} \leq \max _{\pi_{k, g} \neq \pi_{j, g}} \pi_{k g}\right)\right\} .
$$

Note that in order to induce some competitions among sellers, the last condition in the definition of $\mathcal{S}_{g}(\pi)$ implies that the highest bidding seller is excluded from the market (in case the strategy profile is not $p$-uniform). We then define the extended demand of agent $i$ as $\delta_{i}(\pi, w)=d_{i}\left(\pi_{i}, w\right)$ and assume agent $i$ can raise a positive income only from the market to which he has access. That is:

$$
w_{i g}(\pi)=\left\{\begin{array}{cc}
\pi_{i g} \cdot e_{i g} & \text { if } i \in \mathcal{S}_{h}(\pi) \\
0 & \text { otherwise }
\end{array}\right.
$$

Our bargaining set then is the set of price-feasible allocations defined as follows.

Definition 2. The set of price feasible allocations $\mathcal{A}(e, \pi)$ is the subset of feasible allocations $\mathcal{A}(e)$ such that for every $x \in \mathcal{A}(e, \pi)$ we have:

1. $\forall(i, h) \in A \times C_{i}, x_{i h}>0 \Rightarrow i \in \mathcal{B}_{h}(\pi)$;

2. $\sum_{i \in \mathcal{B}_{h}(\pi)} x_{i h} \leq \sum_{j \in \mathcal{S}_{h}(\pi)} e_{j h}$;

3. $\pi_{i} \cdot x_{i} \leq w_{i}(\pi)$.

The set $\mathcal{A}(e, \pi)$ is always non-empty because it contains the zero allocation where for each agent $i$, one has $x_{i}=0$. Now, a feasible allocation is price feasible if it is such that (1) only admissible buyers receive a positive allocation, (2) trade 
takes place only between admissible buyers and sellers, (3) each agent satisfies its private budget constraint. More broadly, Definition 2 expresses the fact that as agents agree on the valuation of goods, i.e as private prices become more similar, the number of successful transactions grows. In particular when a $p$-uniform price profile is reached, then the market includes each agent, and the constraint his private price place on a given agent is simply his private budget constraint.

Agents will thus have an incentive to agree on a common price if they actually gain from an increased number of successful transactions. To express this idea formally, let us consider an exchange mechanism $\xi^{\text {barg }}$ such that for all $\pi \in \Pi$, $\xi^{\text {barg }}(\pi) \in \mathcal{A}(e, \pi)$. Then, agents will gain from an increase in the market's size (provided his budget constraint is not being strengthened) if the exchange mechanism $\xi^{\text {barg }}$ satisfies the monotonicity condition that is standard in the bargaining literature since the seminal paper by Kalai and Smorodinsky 1975, that is:

Assumption 11 (KS-Monotonicity). If $\pi$ and $\pi^{\prime}$ are such that $\mathcal{A}(e, \pi) \subset \mathcal{A}\left(e, \pi^{\prime}\right)$ then for all $i=1 \cdots n$, we have $u_{i}\left(\xi_{i}^{\text {barg }}\left(\pi^{\prime}\right)\right) \geq u_{i}\left(\xi_{i}^{\text {barg }}(\pi)\right)$.

It is then clear from the definitions of $\delta_{i}(\pi, w), w_{i g}(\pi)$ and $\mathcal{A}(e, \pi)$ that the Price Consistency Assumption (4) and the Price Compatibility Assumption (6) hold. Moreover, the Seller Competition Assumption (8) holds for $\xi^{\text {barg }}$ because the most expensive seller is excluded of the market according to the definition of $\mathcal{S}_{h}(\pi)$ and hence he can always increase his income by decreasing his price. Finally, the Income Monotonicity Assumption (9), and the Homogeneous Pricing Assumption (10) as well as the second part of the Equilibrium Assumption (5) are clear consequences of the KS-Monotonicity Assumption (11). In particular, the second condition in the Equilibrium Assumption (5) holds because of the private price constraint in the definition of the set of price-feasible allocations. Thus, using Theorem 1, we have:

Corollary 3. Let $\xi^{\text {barg }}$ be such that for all $\pi \in P i$, $\xi^{\text {barg }}(\pi) \in \mathcal{A}(e, \pi)$, the Equilibrium Assumption (5), the Buyer Competition Assumption (7), and the KSMonotonicity Assumption (11) hold. Then $\pi$ is a strict Nash equilibrium of the game $\mathcal{G}\left(u, e, \xi^{\text {barg }}\right)$ if and only if it is a general market equilibrium price profile.

In other words, for bargaining exchange mechanisms, the monotonicity condition suffices to guarantee that agents have an incentive to agree on a common price. It then suffices to assume there is competition among buyers and that the allocations at a general equilibrium are efficient in order to ensure the stability of general equilibrium price profiles. 


\section{The Scarf Economy}

The examples introduced in the preceding section show that Theorem 1 can be applied in a wide variety of settings. Still, these examples were required to be highly stylized to prove stability. In this section, we investigate numerically whether our results extend to more complex models of individual behavior. More precisely, we introduce a Markovian model of price adjustment, for which the necessary conditions of Theorem 1 do not necessarily hold but which retain the key features of our model: prices are set in a decentralized manner and updated according to an evolutionary mechanism. In this setting, we give computer simulation evidence of convergence to general equilibrium.

We place ourselves in the Scarf (1960) economy, a well-known example of instability of the tâtonnement. Namely, we consider an economy with three goods $(n=3)$ and three types of agents $(m=3)$. Agents of type 1 have utility $u_{1}\left(x_{1}, x_{2}, x_{3}\right)=\min \left(x_{1} / \omega_{1}, x_{2} / \omega_{2}\right)$, and initial endowment $\left(\omega_{1}, 0,0\right)$, agents of type 2 have utility $u_{2}\left(x_{1}, x_{2}, x_{3}\right)=\min \left(x_{2} / \omega_{2}, x_{3} / \omega_{3}\right)$ and initial endowment $\left(0, \omega_{2}, 0\right)$ and agents of type 3 have utility $u_{3}\left(x_{1}, x_{2}, x_{3}\right)=\min \left(x_{3} / \omega_{3}, x_{1} / \omega_{1}\right)$ and initial endowment $\left(0,0, \omega_{3}\right)$. We let $\left(\omega_{1}, \omega_{2}, \omega_{3}\right)=(10,20,400)$ so that equilibrium relative prices, which must be proportional to $p^{*}=(40,20,1)$, are extremely unequal (see Anderson et al. 2004) .

In order to simulate price dynamics in this setting, we consider 1000 agents of each type. Each agent is initially equipped with a private price vector and with an inventory of goods equal to its initial endowment. ${ }^{7}$ Every period, agents engage in a trading process whose outcome depends on the distribution of private prices. This first process can be seen as a dynamic version of the exchange mechanisms introduced in the preceding section and allows to relate the utility gained by each agent to its private price. Every ten periods, ${ }^{8}$ prices are updated according to an evolutionary mechanism where the average utility obtained is used as a measure of fitness of the private price used by the agent.

The trading process is implemented as follows. At the beginning of each period, the inventory of each agent is reset to the value of its initial endowment. Each agent, in random order, is then designated a trade initiator and is paired with a randomly chosen responder, who can either accept or reject the proposed trade. Each

\footnotetext{
${ }^{7}$ More precisely, we assume that there is a smallest possible positive price, and each component of an agent's price vector is drawn from the uniform distribution on the multiples of this smallest possible price in the interval $(0,1)$.

${ }^{8}$ This periodicity can be changed without modifying qualitatively the results. Considering a larger number of periods allows a better approximation of the expected utility of each agent in the trading process.
} 
agent is thus an initiator exactly once and a responder on average once per period. ${ }^{9}$

In order to define the outcome of an individual trade, we have to introduce the following classification of goods. For an agent of type $i$, the good $i$ is called his production good, the additional good that he consumes $\gamma_{i}=\bmod (i+1,3)$ is called his consumption good and the $\operatorname{good} \tau_{i}=\bmod (i+1,2)$ that he neither produces nor consumes is called his transaction good. ${ }^{10}$ Let us then consider the initiator $a$ is an agent of type $i_{a}$, with private price $p_{a} \in \mathbb{R}_{+}^{3}$ and stock of goods $x_{a} \in \mathbb{R}_{+}^{3}$ while the responder has private price $p_{b} \in \mathbb{R}_{+}^{3}$ and stock of goods $x_{b} \in \mathbb{R}_{+}^{3}$. In this setting, a trade of good $k$ against good $\ell$ is acceptable if the trade ratio proposed by the initiator is profitable according to the responder and if the initiator and responder indeed have the corresponding goods in stock, that is:

$$
\frac{p_{a, \ell}}{p_{a, k}} \geq \frac{p_{b, \ell}}{p_{b, k}} \wedge x_{a, k}>0 \wedge x_{b, \ell}>0 .
$$

The initiator $a$ then proposes transactions in the following order:

$$
\left(\tau_{i_{a}}, \gamma_{i_{a}}\right) \succ\left(i_{a}, \gamma_{i_{a}}\right) \succ\left(i_{a}, \tau_{i_{a}}\right)
$$

That is the initiator tries first to trade his transaction good against his consumption good, second his production good against his consumption good and third his production good against his transaction good. The transaction that actually gets executed is the first one that is acceptable in the sense of condition (2). The amounts the initiator proposes to depend on the type of the transaction:

- In a $\left(\tau_{i_{a}}, \gamma_{i_{a}}\right)$ transaction, the initiator proposes his whole stock of transaction good $x_{\tau_{i_{a}}}$ against $p_{\tau_{i_{a}}} x_{\tau_{i_{a}}} / p_{\gamma_{i_{a}}}$ units of his consumption good.

- In a $\left(i_{a}, \gamma_{i_{a}}\right)$ transaction, the initiator bases his request on its utility maximizing demand $d_{i_{a}}\left(p_{a}, p_{a, i_{a}} \cdot x_{a, i_{a}}\right)$ and proposes to trade $x_{i_{a}}-d_{i_{a}, i_{a}}\left(p_{a}, p_{a, i_{a}} \cdot\right.$ $\left.x_{a, i_{a}}\right)$ units of his production good against $d_{i_{a}, i_{b}}\left(p_{a}, p_{a, i_{a}} \cdot x_{a, i_{a}}\right)$ units of his consumption good.

- In a $\left(i_{a}, \tau_{i_{a}}\right)$ transaction, the initiator tries to split his inventory equally between production and transaction goods. Therefore he proposes to trade half his stock of production good, that is, $x_{a, i_{a}} / 2$ units, against $p_{a, i_{a}} x_{a, i_{a}} / 2 p_{a, \tau_{i a}}$ units of his transaction good.

\footnotetext{
${ }^{9}$ Our results are not qualitatively modified if the number of trading opportunities per period is increased.

${ }^{10}$ We denote by $\bmod (u, v)$ the rest in the Euclidian division of $u$ by $v$.
} 
After a successful trade, each agent consumes whatever is feasible from his inventory, i.e he consumes the share of goods he holds in the required proportions $\left(\omega_{i}, \omega_{\gamma_{i}}\right)$. His utility is updated accordingly.

Hence, trading behavior is based on simple rules of thumb and clearly not optimal. However this lack of optimality is perfectly in line with the requirement put forward in Section (4.1) that exchanged mechanisms should not be too efficient out of equilibrium. As a matter of facts, simulation results (see below) show that despite (or because of) the simplicity of the exchange mechanism, the feedback effect utility has on the evolution of private prices suffices to coordinate agents on equilibrium.

This feedback effect is implemented through a stochastic imitation model à la Schlag (1998) for which we use as measure of fitness the average utility of consumption over the previous ten periods. Every ten periods, $5 \%$ of agents are randomly chosen to review their strategy, i.e their vector of private prices. Reviewing agents are matched with randomly selected partners of the same type and the least successful of the pair then copies the private prices of the more successful. In addition to this imitation process, there is a mutation process in which $1 \%$ of the agents are selected and randomly increase or decrease their prices by $10 \%$. It is well known that this mutation process renders the evolution of strategies ergodic (see Fudenberg and Levine, 1997). Moreover, it can be shown (see Benaim and Weibull, 2003) that in the large-population limit, stochastic imitation models are well approximated by the replicator dynamic. Thus, the results of Section 3 suggest that we should observe convergence of prices to their equilibrium values.

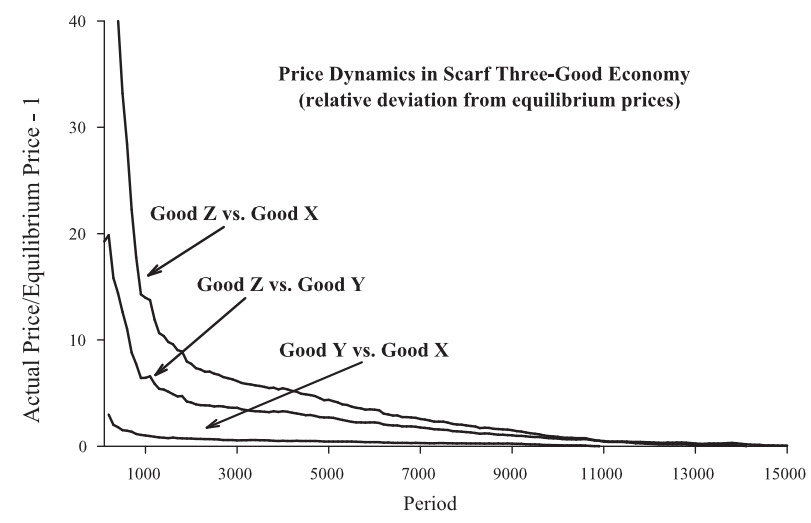

Figure 1: Convergence of Price to Equilibrium in a Three-good Scarf Economy with Private Prices 
The results of a typical run are exhibited in Figure 1. Each of the curves in Figure 1 is given by $\left(p^{*}-p\right) / p^{*}$ where $p^{*}$ is the equilibrium relative price. Because initial prices are generated by uniform distributions on the unit interval, the initial mean price of good 3 is about 40 times its equilibrium value, and the price of good 2 is about 20 times its equilibrium value. Nevertheless, in sharp contrast to the oscillatory behavior of the tâtonnement process in this setting Scarf (1960), the system settles rapidly in a steady state where prices assume their equilibrium values. That is, we observe convergence to general equilibrium.

Similar convergence results have been obtained in economies with Leontieff preferences (see Gintis 2012) as well as in arbitrarily large economies with nested, arbitrarily heterogeneous, CES utility functions where fitness is measured by traded volumes rather than by utility (Gintis 2013). These results suggest that in a setting where agents use private prices as strategies, evolutionary dynamics based on imitation of success (Schlag, 1998; Benaim and Weibull, 2003) can provide dynamical foundations for general market equilibrium in settings where the tâtonnement mechanism fails.

\section{Conclusion}

We have introduced a new class of market games in which agents use private prices as strategies. In this framework, provided the exchange process implements some form of competition both between buyers and between sellers, strict Nash equilibria coincide with general market equilibria. This result has two main corollaries. First, it implies that competitive equilibria can be strategically stable even in small economies. Second, it implies that competitive equilibria have good local stability properties under a large class of evolutionary dynamics.

The major innovation of our model is the use of private prices, one set for each agent, in place of the standard assumption of a uniform public price faced by all agents. The private price assumption is a natural assumption for a fully decentralized market system not in equilibrium, because there is in fact no natural way to define a common price system except in equilibrium. With private prices, each individual is free to alter his private prices at will, market conditions alone ensuring that something approximating a uniform system of prices will prevail in the long run.

Our results suggest that a micro-economic theory of the formation of prices based on evolutionary processes is worth considering. Such an approach can in particular be used to develop micro-founded models of macro-economic dynamics that account for disequilibrium and the endogenous emergence of aggregate volatility (see Mandel et al., 2015, for preliminary work in that direction). 


\section{References}

Alós-Ferrer, C., Ania, A. B., Schenk-Hoppé, K. R., 2000. An evolutionary model of bertrand oligopoly. Games and Economic Behavior 33 (1), 1-19.

Anderson, C. M., Plott, C. L., Shimomura, K.-I., Granat, S., 2004. Global instability in experimental general equilibrium: The scarf example. Journal of Economic Theory 115, 209-249.

Asparouhova, E., Bossaerts, P., Ledyard, J. O., 2011. Price formation in continuous double auctions; with implications for finance, citeseer.

Balasko, Y., 2009. The Equilibrium Manifold. Postmodern Developments in the Theory of General Economic Equilibrium. MIT Press, Cambridge.

Benaim, M., Weibull, J. W., 2003. Deterministic approximation of stochastic evolution in games. Econometrica 71 (3), 873-903.

Dockès, P., Potier, J.-P., 2005. Léon walras et le statut de la concurrence: une étude à partir des elements d'économie politique pure. Histoire des représentations du marché, 366-391.

Duffy, J., Matros, A., Temzelides, T., 2011. Competitive behavior in market games: Evidence and theory. Journal of Economic Theory 146 (4), 1437-1463.

Easley, D., Ledyard, J., 1993. Theories of price formation and exchange in double oral auctions. The double auction market: Institutions, theories, and evidence, 63-97.

Florenzano, M., 2005. General Equilibrium Analysis: Existence and Optimality Properties of Equilibria. Springer, Berlin.

Friedman, D., 1991. A simple testable model of double auction markets. Journal of Economic Behavior \& Organization 15 (1), 47-70.

Fudenberg, D., Levine, D. K., 1997. The Theory of Learning in Games. MIT Press, Cambridge, MA.

Gale, D., Jul. 1986a. Bargaining and competition, part i: Characterization. Econometrica 54 (4), 785-806.

Gale, D., Jul. 1986b. Bargaining and competition, part ii: Existence. Econometrica $54(4), 807-818$. 
Ghosal, S., Morelli, M., 2004. Retrading in market games. Journal of Economic Theory 115 (1), 151-181.

Gintis, H., 2007. The dynamics of general equilibrium*. The Economic Journal 117 (523), 1280-1309.

Gintis, H., 2009. Game Theory Evolving Second Edition. Princeton University Press, Princeton.

Gintis, H., 2012. The dynamics of pure market exchange. In: Aoki, M., Binmore, K., Deakin, S., Gintis, H. (Eds.), Complexity and Institutions: Norms and Corporations. Palgrave, London.

Gintis, H., Jun. 2013. Markov models of social exchange: Theory and applications. ACM Transactions in Intelligent Systems and Technology $4(3,53)$.

Giraud, G., 2003. Strategic market games: An introduction. Journal of Mathematical Economics 39, 355-375.

Gjerstad, S., Dickhaut, J., 1998. Price formation in double auctions. Games and economic behavior 22 (1), 1-29.

Grandmont, J. M., 1977. Temporary general equilibrium theory. Econometrica, $535-572$.

Hammond, P. J., 1993. Irreducibility, resource relatedness and survival in equilibrium with individual nonconvexities in general equilibrium. In: Becker, R., Boldrin, M., Jones, R., Thomson, W. (Eds.), Growth and Trade. Academic Press, San Diego, pp. 73-115.

Hopkins, E., 2002. Two competing models of how people learn in games. Econometrica 70 (6), 2141-2166.

Ianni, A., 2013. Learning strict nash equilibria through reinforcement. Journal of Mathematical Economics.

Kalai, E., Smorodinsky, M., May 1975. Other solutions to nash's bargaining problem. Econometrica 43 (3), 513-518.

Kim, C., Wong, K.-C., 2011. Evolution of walrasian equilibrium in an exchange economy. journal of evolutionary economics 21 (4), 619-647.

Kumar, A., Shubik, M., 2004. Variations on the theme of scarf's counter-example. Computational Economics 24 (1), 1-19. 
Kunimoto, T., Serrano, R., 2002. Bargaining and competition revisited, brown University Working Paper 2002-14.

Lesourne, J., Orléan, A., Walliser, B., 2006. Evolutionary microeconomics. Springer Science \& Business Media.

Mandel, A., Botta, N., 2009. A note on herbert gintis' emergence of a price system from decentralized bilateral exchange. The B.E. Journal of Theoretical Economics 9 (1).

Mandel, A., Gintis, H., 2014. Stochastic stability in the scarf economy. Mathematical Social Sciences 67 (0), $44-49$.

URL http://www.sciencedirect.com/science/article/pii/S0165489613000796

Mandel, A., Landini, S., Gallegati, M., Gintis, H., 2015. Price dynamics, financial fragility and aggregate volatility. Journal of Economic Dynamics and Control 51, 257-277.

Mas-Collel, A., 1989. The Theory of General Economic Equilibrium: A Differentiable Approach. Cambridge University Press, Cambridge.

McLennan, A., Sonnenschein, H., 1991. Sequential bargaining as a noncooperative foundation of competitive equilibrium. Econometrica 59, 13951421.

Peck, J., Shell, K., 1990. Liquid markets and competition. Games and Economic Behavior 2 (4), 362-377.

Rubinstein, A., 1982. Perfect equilibrium in a bargaining model. Econometrica 50, 97-109.

Rubinstein, A., Wolinsky, A., 1985. Equilibrium in a market with sequential bargaining. Econometrica 53, 1133-1150.

Scarf, H., 1960. Some examples of global instability of competitive equilibrium. International Economic Review 1, 157-172.

Schlag, K., 1998. Why imitate, and if so, how? a boundedly rational approach to multi-arm bandits. Journal of Economic Theory 78, 130-156.

Serrano, R., Volij, O., 2008. Mistakes in cooperation: the stochastic stability of edgeworth's recontracting. Economic Journal 118, 1719-1741.

Shalizi, C., 2009. Dynamics of bayesian updating with dependent data and misspecified model. Electronic Journal of Statistics 3, 1039-10-74. 
Shapley, L., Shubik, M., 1977. Trade using one commodity as a means of payment. Journal of Political Economy 85, 937-968.

Smith, V., 1982. Microeconomic systems as an experimental science. American Economic Review, 923-955.

Vega-Redondo, F., 1997. The evolution of walrasian behavior. Econometrica 61, $57-84$.

Walras, L., 1874. Elements of Pure Economics. George Allen and Unwin, London.

Weibull, J. W., 1995. Evolutionary Game Theory. MIT Press, Cambridge, MA. 